\title{
The efficacy of third generation anti-HER2 chimeric antigen receptor $T$ cells in combination with PD1 blockade against malignant glioblastoma cells
}

\author{
LUXI SHEN ${ }^{1}$, HONGZHI LI ${ }^{2}$, SHUFANG BIN ${ }^{2}$, PANYUAN LI ${ }^{2}$, JIE CHEN ${ }^{2}$, \\ HAIHUA GU ${ }^{2}$ and WEIHUA YUAN ${ }^{2}$

\begin{abstract}
${ }^{1}$ Beijing Friendship Hospital, Capital Medical University, Beijing $100050 ;{ }^{2}$ Key Laboratory of Laboratory Medicine, Ministry of Education, Zhejiang Provincial Key Laboratory of Medical Genetics, School of Laboratory Medicine and Life Sciences, Wenzhou Medical University, Wenzhou, Zhejiang 325035, P.R. China
\end{abstract}

Received January 25, 2019; Accepted June 14, 2019

DOI: 10.3892/or.2019.7263

\begin{abstract}
Without effective treatment, glioblastoma is one of the deadliest cancers worldwide. The aim of the present study was to explore whether combinational immunotherapy is effective for treating malignant glioblastoma in vitro. The therapeutic efficacy of third generation anti-human epidermal grow th factor receptor 2 (HER2) chimeric antigen receptor (CAR)-T cells alone and in combination with PD1 blockade was investigated for the treatment of malignant glioblastoma cells in vitro. Anti-HER2 CAR-T cells were prepared by transducing activated primary human T cells with lentiviruses which expressed third generation anti-HER2 CAR. The CAR-positive cell ratio was detected using flow cytometry. The expression level of CAR was detected by western blot analysis. The binding of anti-HER2 CAR-T cells to HER2 ${ }^{+}$U251 glioblastoma cells was examined under a fluorescence microscope. The cytokine
\end{abstract}

Correspondence to: Dr Luxi Shen, Beijing Friendship Hospital, Capital Medical University, 95 Yongan Road, Beijing 100050, P.R. China

E-mail: imaginary-diva@163.com

Dr Weihua Yuan, Key Laboratory of Laboratory Medicine, Ministry of Education, Zhejiang Provincial Key Laboratory of Medical Genetics, School of Laboratory Medicine and Life Sciences, Wenzhou Medical University, North Zhongshan Road, Wenzhou, Zhejiang 325035, P.R. China

E-mail: ywh038943@163.com

Abbreviations: CAR, chimeric antigen receptor; PD1, programmed death-1; ATCC, American Tissue Culture Collection; DMEM, Dulbecco's modified Eagle's medium; FBS, fetal bovine serum; PBMCs, peripheral blood mononuclear cells; IL-2, interleukin-2; IFN- $\gamma$, interferon- $\gamma$; MOI, multiplicity of infection; LDH, lactate dehydrogenase; 7-AAD, 7-aminoactinomycin D; TIL, tumor-infiltrating lymphocyte

Key words: HER2, chimeric antigen receptor, T cells, PD1, glioblastoma secretion of CAR-T cells induced by target cells was analyzed via ELISA. The cytotoxicity of anti-HER2 CAR-T cells alone or in combination with anti-programmed death-1 (PD1) antibody against $\mathrm{HER} 2^{+} / \mathrm{PDL}^{+} \mathrm{U} 251$ cells was examined using an LDH assay. The CAR-positive cell ratio and expression level of CAR in prepared CAR-T cells were both high enough. Anti-HER2 CAR-T cells could specifically bind to U251 cells. The IL-2 and IFN- $\gamma$ secretion of CAR-T cells increased after being co-cultured with U251 cells, and further increased in the presence of anti-PD1 antibody. Anti-HER2 CAR-T cells displayed a potent cytotoxicity against U251 cells. In addition, the presence of anti-PD1 antibody further enhanced the efficacy of anti-HER2 CAR-T cells against U251 cells. The present results indicated that blocking PD1 immuno-suppression can increase the activation of CAR-T cells after they are activated by a targeting antigen. Third generation anti-HER2 CAR-T cells along with PD1 blockade have a great therapeutic potential for combatting malignant glioblastoma.

\section{Introduction}

Glioblastoma is the deadliest primary central nervous system tumor and has a prevalence of $3.19 \%$ in the United States (1). In the past 15 years, treatment of glioblastoma has included maximal safe surgical resection combined with radiotherapy and temozolomide chemotherapy (2). Despite this, the overall five-year survival is still less than 5\%, with an average survival of just 14 months following initial diagnosis (3). Immunotherapy is an alternative approach which has the potential to overcome the limitations of the current standard therapies. In the past five years, chimeric antigen receptor (CAR)-T immunotherapy has had substantial success in the treatment of chronic lymphocytic leukemia and acute lymphoblastic leukemia patients who have relapsed from chemotherapy treatment (4-6).

The CAR-T immunotherapy method uses genetically engineered $\mathrm{T}$ cells to express CAR. First generation CAR is composed of an antigen recognition domain ( $\mathrm{scFv}$ ) and the essential activating signal $\mathrm{CD} 3 \zeta$, while second generation CAR includes one co-stimulatory molecule and the third generation includes two co-stimulatory molecules (7). 
The antigen recognition domain confers a specificity of CAR-T cells for tumor-associated antigens. Thus, finding a suitable tumor antigen is important for the use of CAR-T cells to treat glioblastoma. Human epidermal growth factor receptor 2 (HER2), a member of the EGFR family, encodes a $185-\mathrm{kDa}$ transmembrane protein via tyrosine protein kinase activity. The gene amplification and protein overexpression of HER2 in human tumors have been associated with more aggressive cancers $(8,9)$. The HER 2 antigen is overexpressed in approximately $25-30 \%$ of patients living with breast cancer, $30-40 \%$ of patients with primary renal cell carcinoma and $30-35 \%$ of patients with lung adenocarcinoma (10-12). HER2 expression is absent in the adult central nervous system (13). CAR-T cells targeting HER 2 have been demonstrated to be safe and effective in clinical trials $(14,15)$.

According to previous studies, HER2 is expressed in as many as $80 \%$ of glioblastoma cases $(16,17)$. Furthermore, overexpression of HER2 has been revealed to be correlated with the malignancy of glioma (18) and with high-grade glioma (19) as well as with early mortality (9). These data indicate that HER2 is an attractive therapeutic target for glioblastoma. In the preclinical model of glioblastoma, CAR-T cells being studied for glioma-targeted antigens IL-13R $\alpha 2$, EphA2, HER2 (using a second generation anti-HER2 CAR) and EGFRvIII (20) demonstrated strong anti-tumor activity. In addition to careful selection of the tumor-associated antigen, the optimal design of the CAR architecture (such as using a new generation anti-HER2 CAR) is an important prerequisite for achieving significant responses in CAR-mediated immunotherapy.

While CAR-T cells have exhibited significant responses in intractable hematological malignancies, they have low therapeutic effects for solid tumors. This may be due to the many obstacles in the tumor microenvironment of solid tumors (21-26), such as the intrinsic inhibition pathway mediated by $\mathrm{T}$ cell surface inhibitory receptors binding to their tumor cell surface ligands (26). One of the most frequently studied $\mathrm{T}$ cell inhibitory receptors is programmed death-1 (PD1/CD279), which is upregulated following the engagement of $\mathrm{T}$ cell receptor with its ligand. Currently, the known ligand of PD1 in various cancers is programmed cell death 1 ligand 1 (PDL1/CD274) (27). However, PDL1 on the cell surface of solid tumors is typically upregulated in response to cytokines (such as IL-2 and IFN- $\gamma$ ) which have been secreted by $\mathrm{T}$ cells, thus serving as tumor immune evasion (24).

In the present study, a third generation anti-HER2 CAR (anti-HER2 scFv-CD28-CD137-CD3ל) was used, which we previously designed and constructed, and which carried two co-stimulatory molecules (CD28 and CD137). The third generation anti-HER2 CAR has not been reported in clinical trials for treatment of malignant glioblastoma. Lentivirus-mediated $\mathrm{T}$ cell transduction was utilized to generate CAR-T cells. It was examined whether anti-HER2 CAR-T cells alone have specific and efficient cytotoxicity, as well as whether their combination with PD1 blockade enhances the therapeutic activity against $\mathrm{HER} 2^{+} / \mathrm{PDL1}^{+}$glioblastoma cells in vitro. The use of anti-HER2 CAR-T cells in combination with anti-PD1 antibody has not been reported in clinical trials for malignant glioma.

\section{Materials and methods}

Cell lines and media. 293T-17 cells [American Tissue Culture Collection (ATCC)], human malignant glioblastoma cells U87 (glioblastoma of unknown origin, HTB-14 ${ }^{\mathrm{TM}}$ ) (ATCC) as well as U251 (China Center for Type Culture Collection) were cultured in Dulbecco's Modified Eagle's Medium (DMEM) (Gibco; Thermo Fisher Scientific, Inc.) and supplemented with $10 \%$ fetal bovine serum (FBS) (both from Gibco; Thermo Fisher Scientific, Inc.). The U251 cell line was authenticated using STR analysis (Cell Bank, Type Culture Collection, Chinese Academy of Sciences).

Construction of anti-HER2 CAR and production of recombinant lentivirus. The third generation anti-HER2 CAR was developed by our research group and synthesized by the Beijing Genomics Institute in China. The recombinant lentivirus was produced using 293T-17 cells co-transfected with recombinant lentiviral vector $\mathrm{pLVX}$-EF1 $\alpha$-CAR-IRES-ZsGreen1 as well as packaging plasmids psPAX2 and pMD2.G. Packaging, concentration and purification of the recombinant lentivirus were performed following protocols we had optimized (28).

Preparation of human peripheral T lymphocytes. Preparation of human peripheral T lymphocytes, including isolation, activation, and purification, was performed following the protocols we have previously described $(28,29)$ but with some further optimization. The present study was approved by the Ethical Committee of Wenzhou Medical University (Wenzhou, China). Peripheral blood materials used in this study were obtained from healthy donors who provided informed consent. The total number of healthy donors used was three (including one male and two females), the age distribution was 24-28, collected from January 2017 to December 2018 at the First Affiliated Hospital of Wenzhou Medical University. Peripheral blood mononuclear cells (PBMCs) were isolated from heparinized peripheral blood by density-gradient centrifugation (at $900 \mathrm{x} \mathrm{g}$ ) utilizing a lymphocyte separation medium (Sigma-Aldrich; Merck KGaA). PBMCs were activated (stimulated) using anti-CD2-, anti-CD3- and anti-CD28-coated microbeads (Miltenyi Biotec $\mathrm{GmbH}$ ) at a 1:1 bead to cell ratio for two days. Primary human peripheral T lymphocytes were cultured in GT-T551 medium (Takara Bio, Inc.) which had been supplemented with $10 \%$ FBS and 300 IU/ml Interleukin-2 (IL-2) (PeproTech, Inc.).

Transduction and expansion of $T$ cells. The $\mathrm{CD}^{+}$human peripheral $\mathrm{T}$ cells were transduced with recombinant lentivirus at a multiplicity of infection (MOI) value of 20, as previously described (29). For transduction, the cell plate was centrifuged at $1,200 \times \mathrm{g}$ for $2 \mathrm{~h}$ and cultured for $10-14 \mathrm{~h}$, then replaced with fresh medium and cultured for expansion. After examining the proper GFP expression level ( $40 \%$ GFP-positive cell ratio) under fluorescence microscope (Nikon Eclipse Ti), the transduced cells were subjected to flow cytometry and western blotting to evaluate the GFP-positive cell percentage and CAR expression level respectively.

Flow cytometric analysis. The expression of HER2 in glioblastoma cells U87 and U251 was determined using APC 
anti-human CD340 (erbB2/HER2) antibody (cat. no. 324407), with APC mouse IgG1 $\kappa$ (both from BioLegend, Inc.) used as an isotype control. The expression of PDL1 in glioblastoma cells U87 and U251 was evaluated using PE anti-human CD274 (PDL1) antibody (BD Biosciences), while PE mouse IgG2ak (BD Biosciences) was used as an isotype control. Transduction efficiency to $\mathrm{T}$ cells was measured by the percentage of transduced T cells expressing GFP (that is, CAR). Flow cytometric data were analyzed using FlowJo v10 software (FlowJo LLC).

Western blot analysis. Transduced human peripheral $\mathrm{T}$ cells were detected for the expression level of CAR by western blot analysis. T cells were lysed in modified RIPA lysis buffer $[1 \%$ Triton X-100, $50 \mathrm{mM}$ Tris-HCl, $\mathrm{pH}$ 7.4, 1 mM EDTA, $150 \mathrm{mM}$ $\mathrm{NaCl}, 0.25 \%$ Na-deoxycholate, $0.05 \%$ SDS, $10 \mathrm{mM} \mathrm{NaF}, 1 \mathrm{mM}$ sodium vanadate, $1 \mathrm{mM}$ phenylmethylsulfonyl fluoride and protease inhibitor cocktail (Sigma-Aldrich; Merck KGaA)]. The BCA assay kit (Beyotime Institute of Biotechnology) was used to determine the protein concentration. Lysates $(10 \mu \mathrm{g}$ per lane) were resolved by $10 \%$ SDS-PAGE, and transferred to polyvinylidene difluoride (PVDF) membranes (EMD Millipore). The membranes were blocked in TBST containing 5\% non-fat dry milk, immunoblotted with anti-human CD3 $\zeta$ antibody (cat. no. ab226475; dilution 1:1,000; Abcam) and anti-rabbit HRP-conjugated secondary antibody (cat. no. sc-2004; dilution 1:5,000; Santa Cruz Biotechnology, Inc.) and then developed utilizing ECL reagent (Beyotime Institute of Biotechnology). Light emission was detected using the ChemiDoc MP Imaging System (Bio-Rad Laboratories, Inc.). Expression levels of CAR (containing exogenous $\mathrm{CD} 3 \zeta$ ) were detected, using endogenous $\mathrm{CD} 3 \zeta$ as a loading control.

Examination of the targeting ability of CAR-T cells. In order to examine the targeting of anti-HER2 CAR-T cells on HER2-positive glioblastoma cells in vitro, HER2-positive U251cells (or HER2-negative control U87cells) were seeded in a 96-well plate at $1 \times 10^{4}$ cells/well for $6 \mathrm{~h}$. Anti-HER2 CAR-T cells were then added at an E:T (effector to target) ratio of $4: 1$ to co-culture for $24 \mathrm{~h}$. The binding ability of transduced $\mathrm{T}$ cells to target cells as well as the status of target cells were observed using a fluorescence microscope.

Detection of cytokine secretion of CAR-T cells. Anti-HER2 CAR-T cells (effector cells) (or in combination with $20 \mu \mathrm{g} / \mathrm{ml}$ anti-PD1 antibody) were co-cultured with HER2 $2^{+}$glioblastoma cells U251 (target cells) at an E:T ratio of 4:1, for 24,48 or $72 \mathrm{~h}$ in a 96-well plate. Anti-PD1 antibody (clone RMP1-14) was obtained from Bio X Cell. ELISA kits (R\&D Systems, Inc.) for detecting IL-2 and IFN- $\gamma$ were utilized to analyze supernatants of cells according to the manufacturer's instructions. Supernatants from co-culture of anti-HER2 CAR-T cells with HER2- glioblastoma cells U87 as well as from co-culture of (untransduced) blank $\mathrm{T}$ cells (or in combination with anti-PD1 antibody) with HER2 ${ }^{+}$ glioblastoma cells U251 were the negative controls.

Cytotoxicity assay. Anti-HER2 CAR-T cells (effector cells) (or in combination with $20 \mu \mathrm{g} / \mathrm{ml}$ anti-PD1 antibody) were co-cultured with HER $2^{+}$glioblastoma cells U251 (target cells) at E:T ratios of 2:1, 4:1, 8:1 and 16:1 for $18 \mathrm{~h}$ in a 96-well plate. The co-cultures of anti-HER2 CAR-T cells with
HER 2 glioblastoma cells U87 and the blank T cells (or in combination with anti-PD1 antibody) with HER $2^{+}$glioblastoma cells U251 were used as negative controls. Specific lactate dehydrogenase (LDH) which had been released from target cells in cell-free supernatant was detected using a cytotoxicity LDH detection kit (Genmed) according to the manufacturer's instructions. The amount of LDH released was used to assess the lysis of target cells, which may be translated into the effectiveness of effector cells. Percent cytotoxicity was calculated according to OD values utilizing the following formula: Cytotoxicity $(\%)=($ Experimental-Effector spontaneous-Target spontaneous)/(Target maximum-Target spontaneous) x100\%.

Statistical analysis. Probability (P)-values were calculated with GraphPad Prism 5.0 software (GraphPad Software). All experiments were repeated at least three times. Group means were compared via one-way analysis of variance/Newman-Keuls. Differences of $\mathrm{P}<0.05$ were considered to indicate a statistically significant difference.

\section{Results}

Expression of HER 2 and PDL1 in glioblastoma cells. In order to assess the efficacy of anti-HER2 CAR-T cells in combination with anti-PD1 antibody against tumor cells, it is crucial to determine the expression level of HER2 and PDL1 in tumor cells. Flow cytometry was used to detect HER2 and PDL1 expression. The expression levels of HER2 in glioblastoma cells U251 and U87 were 52.6 and $0.55 \%$ respectively (Fig. 1A). The expression levels of PDL1 in glioblastoma cells U251 and U87 were 90.9 and $9.4 \%$ respectively (Fig. 1B). Glioblastoma cell line U251 was set as a HER2 ${ }^{+} / \mathrm{PDL}^{+}$positive target cell, while glioblastoma cell U87 was set as a HER2//PDL1 negative control cell.

Preparation of the third generation anti-HER2 CAR-T cells. The structure of anti-HER2 CAR constructed by our research group is presented in Fig. 2A. Human peripheral T cells were separated and activated. Once human peripheral $\mathrm{T}$ cells had been transduced with recombinant lentivirus at a multiplicity of infection (MOI) of 20 for five days, they were observed under a fluorescence microscope in visible light (Fig. 2B-a) and fluorescence (Fig. 2B-b) to evaluate the percentage of GFP-positive cells. Percentages of viable cells (7-AAD negative cells) and GFP-positive cells (that is, transduction efficiency) were analyzed using flow cytometry (Fig. 2C). Transduction efficiency was $38.2 \%$ (Fig. 2C). The expression levels of CAR (containing exogenous $\mathrm{CD} 3 \xi$ ) were detected utilizing western blotting, using endogenous $\mathrm{CD} 3 \zeta$ as a loading control. CAR-T cells expressed not only endogenous $\mathrm{CD} 3 \zeta(16 \mathrm{kDa})$ but also the expected exogenous $\mathrm{CD} 3 \zeta$ included in CAR (58 kDa) (Fig. 2D).

Targeting of anti-HER2 CAR-T cells to HER2-positive glioblastoma cells. In order to examine the targeting of anti-HER2 scFv on the anti-HER2 CAR-T cell surface, anti-HER2 CAR-T cells were co-cultured with HER2-positive U251 cells for $24 \mathrm{~h}$. The binding ability of anti-HER2 CAR-T cells to U251 cells was examined under a fluorescence microscope in both visible light and fluorescence. CAR-T cells 
A

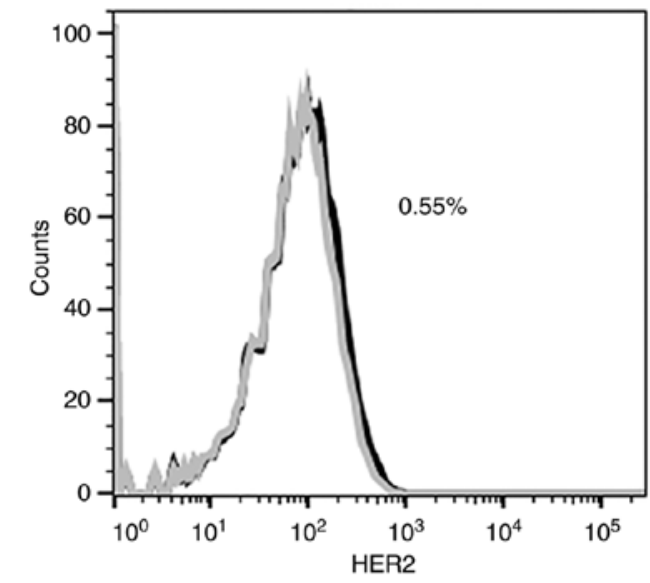

B

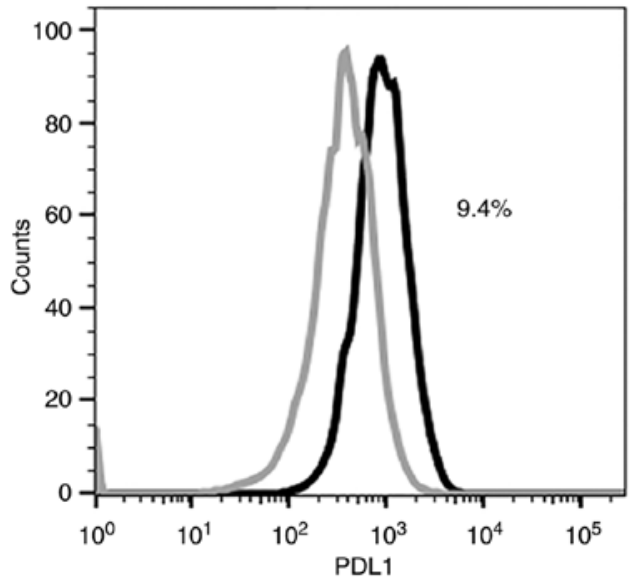

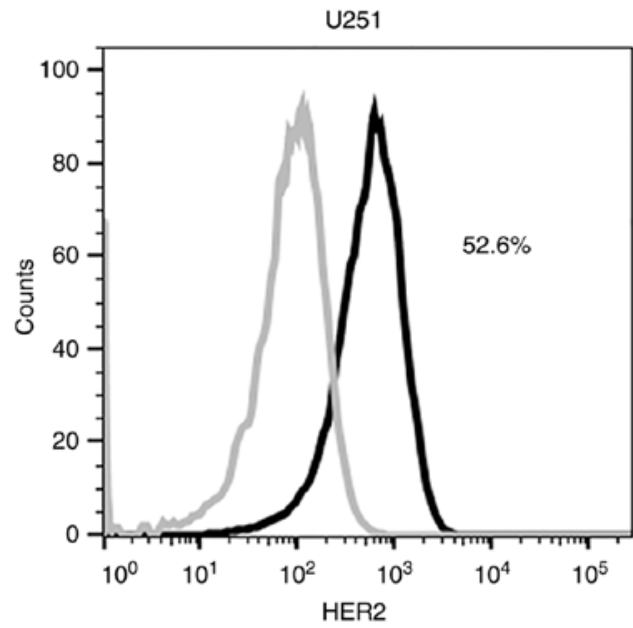

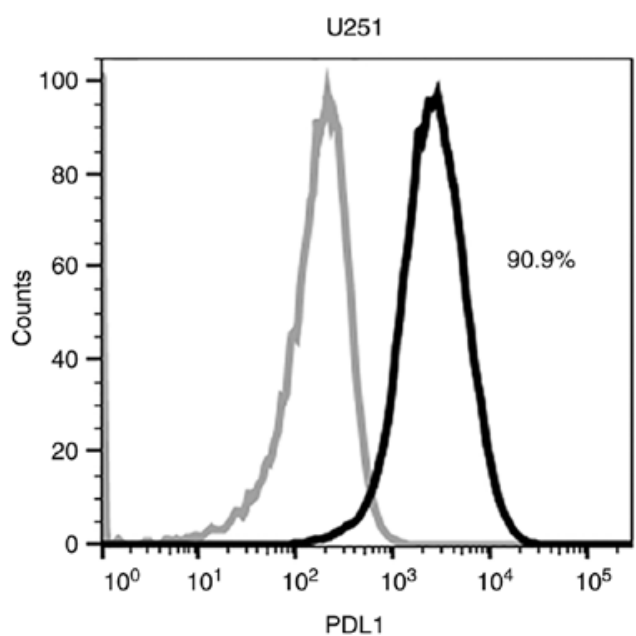

Figure 1. Expression of HER2 and PDL1 in glioblastoma cells. (A) The expression of HER2 in glioblastoma cells U87 and U251 was detected using flow cytometry (using anti-human APC-HER2). The values indicate the expression percentage of HER2. The grey histogram was an isotype control. (B) Expression of PDL1 in glioblastoma cells U87 and U251 was detected utilizing flow cytometry (using anti-human PE-PDL1). The values indicate the expression percentage of PDL1. The grey histogram was an isotype control. HER2, human epidermal growth factor 2; PDL1, programmed cell death 1 ligand 1.

which had been co-cultured with U251 cells formed clusters with U251 cells (Fig. 3C), while the number of viable U251 cells decreased and seemingly disappeared (Fig. 3B) when compared to the number of viable U251 cells not co-cultured with CAR-T cells (Fig. 3A). CAR-T cells did not combine with U87 cells, while most U87 cells remained alive (Fig. 3D-F).

Activation levels of anti-HER2 CAR-T cells induced by HER2-positive glioblastoma cells. In order to determine whether anti-HER2 CAR-T cells alone or in combination with anti-PD1 antibody were activated when co-cultured with target cells, cytokine secretion of anti-HER2 CAR-T cells was detected. The results demonstrated that IL- 2 or IFN- $\gamma$ secretion of the CAR-T group after being co-cultured with the U251 cells for 24, 48 or $72 \mathrm{~h}$ significantly increased when compared to the IL-2 or IFN- $\gamma$ secretion of the blank T group which had been co-cultured with $\mathrm{U} 251$ cells for the same time $(\mathrm{P}<0.01$ or $\mathrm{P}<0.001$ ) (Fig. 4). Secreted IL-2 and IFN- $\gamma$ from the CAR-T group, after being co-cultured with U251 cells for $24 \mathrm{~h}$, increased 27-fold and 15-fold, respectively when compared to those of the blank T group. Secreted IL-2 and IFN- $\gamma$ from the CAR-T group, after being co-cultured with U251 cells for $24 \mathrm{~h}$, increased 5.4-fold and 12.5-fold respectively when compared to those of the CAR-T group which had been co-cultured with U87 cells for $24 \mathrm{~h}$. Notably, following the addition of anti-PD1 antibody, CAR-T cells could significantly increase production of IL-2 and IFN- $\gamma$ after being co-cultured with U251 cells for $72 \mathrm{~h}$, when compared to those of CAR-T cells alone $(\mathrm{P}<0.05$ and $\mathrm{P}<0.01$ ) (Fig. 4). CAR-T cells in combination with anti-PD1 antibody could significantly increase production of IL-2 and IFN- $\gamma$ after being co-cultured with U251 cells for 24,48 or $72 \mathrm{~h}$, when compared to those of blank T cells in combination with anti-PD1 antibody $(\mathrm{P}<0.001)$ (Fig. 4).

Cytotoxicity of anti-HER2 CAR-T cells alone or in combination with anti-PD1 antibody against HER2-positive glioblastoma cells. In order to evaluate the efficacy of effector cells alone or in combination with PD1 blockade on target cells in vitro, anti-HER2 CAR-T cells alone or those in combination with anti-PD1 antibody were co-cultured with HER2-positive glioblastoma cells U251 at E:T ratios of 2:1, 4:1, 8:1 and 16:1 for $18 \mathrm{~h}$. The cytotoxicity (\%) of CAR-T cells to lyse U251 cells was revealed to be significantly higher than that of blank $\mathrm{T}$ cells at every E:T ratio $(\mathrm{P}<0.05$ and $\mathrm{P}<0.001)$ (Fig. $5 \mathrm{~A})$. Notably, the addition of anti-PD1 Ab significantly increased cytotoxicity (\%) of CAR-T cells against U251 cells at the E:T 


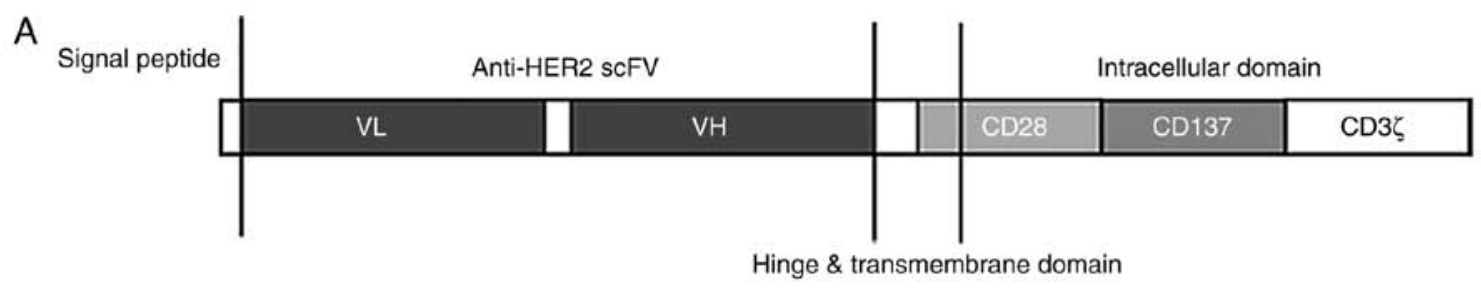

B a

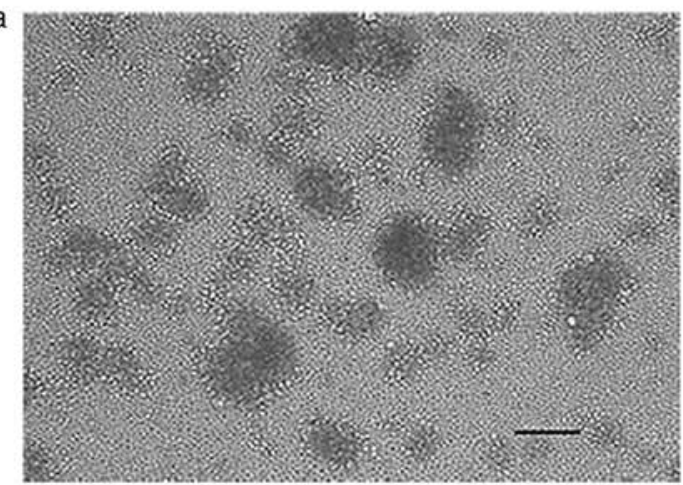

C

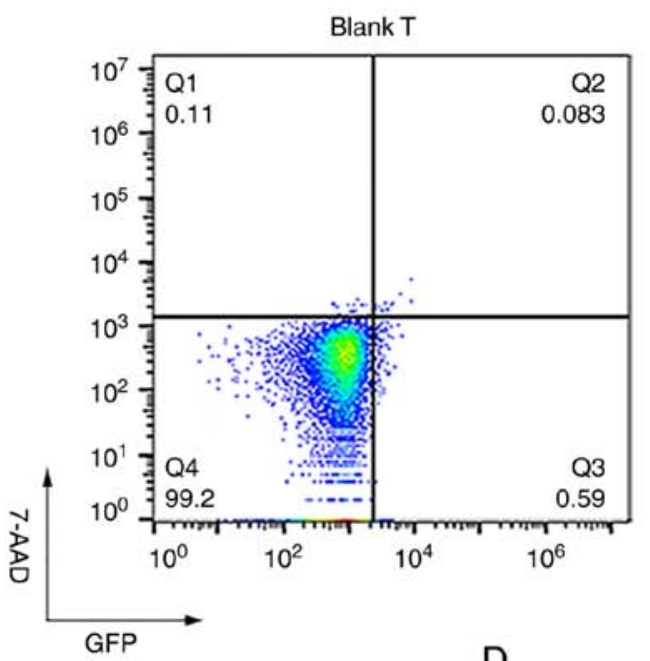

b

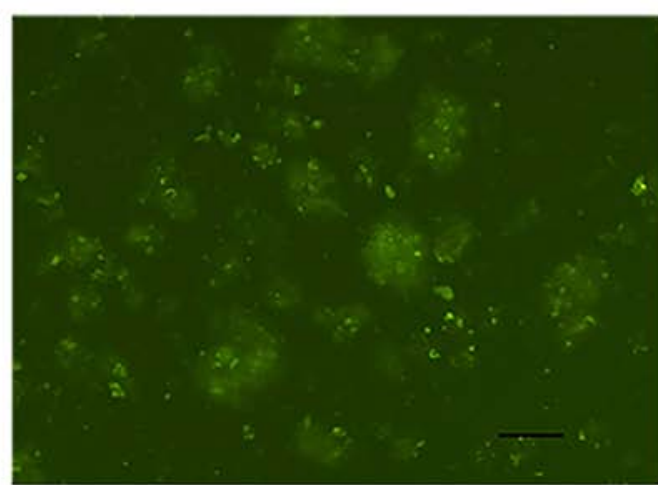

CAR-T

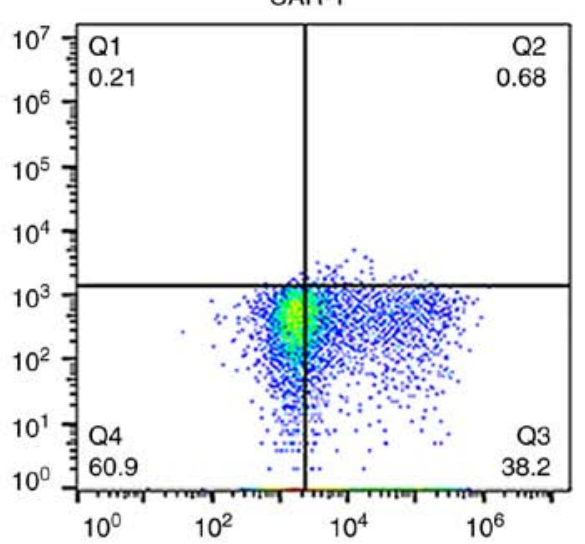

Blank T CAR-T

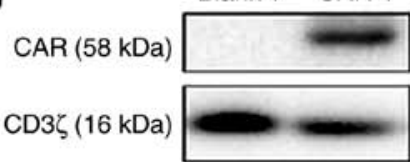

Figure 2. Preparation of the third generation anti-HER2 CAR-T cells. (A) Structure of anti-HER2 CAR. (B) After human peripheral T cells were transduced with recombinant lentivirus at MOI 20 for five days, $T$ cells were observed under a fluorescence microscope in (a) visible light and (b) fluorescence to evaluate the percentage of GFP-positive cells (scale bar, $100 \mu \mathrm{m}$ ). (C) Percentages of viable cells (7-AAD negative cells) and GFP-positive cells (i.e., transduction efficiency) were analyzed using flow cytometry after the human peripheral $\mathrm{T}$ cells were transduced with recombinant lentivirus at MOI 20 for five days.

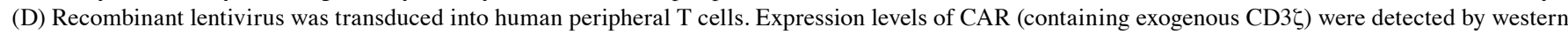
blotting, using endogenous $\mathrm{CD} 3 \zeta$ as a loading control. HER2, human epidermal growth factor 2; CAR, chimeric antigen receptor; MOI, multiplicity of infection; scFv, single chain variable fragment; $\mathrm{VL}$, light chain variable region; $\mathrm{VH}$, heavy chain variable region; 7-AAD, 7-aminoactinomycin D; Blank T, T cells not transduced; CAR-T, T cells transduced with CAR.

ratios of 8:1 and 16:1 compared to that of CAR-T cells alone $(\mathrm{P}<0.05$ and $\mathrm{P}<0.01)$ (Fig. 5A). CAR-T cells in combination with anti-PD1 antibody could significantly increase cytotoxicity (\%) against U251 cells at the E:T ratios of 2:1, 4:1, 8:1 and 16:1, when compared to that of blank $T$ cells in combination with anti-PD1 antibody $(\mathrm{P}<0.05, \mathrm{P}<0.01$ and $\mathrm{P}<0.001)$ (Fig. 5A). As a negative control, anti-HER2 CAR-T cells were co-cultured with HER2 glioblastoma cells U87 (Fig. 5B). At an E:T of 16:1, cytotoxicity (\%) of CAR-T cells against U251 cells reached $46.65 \%$, while cytotoxicity (\%) against HER2-negtive U87 cells was much lower, at just $15.74 \%$ (Fig. 5C) $(\mathrm{P}<0.01)$.
Furthermore, at the E:T ratio of 16:1, following the addition of anti-PD1 Ab, cytotoxicity (\%) of CAR-T cells against U251 cells reached $63.08 \%$, while cytotoxicity (\%) against U87 cells remained low at $17.76 \%$, meaning the former was significantly higher (Fig. 5C) $(\mathrm{P}<0.01)$.

\section{Discussion}

The anti-HER2 CAR developed by our group contains CD28-CD137-CD36, which belongs to third-generation CAR (Fig. 2A). In a reported preclinical study for glioblastoma, the 

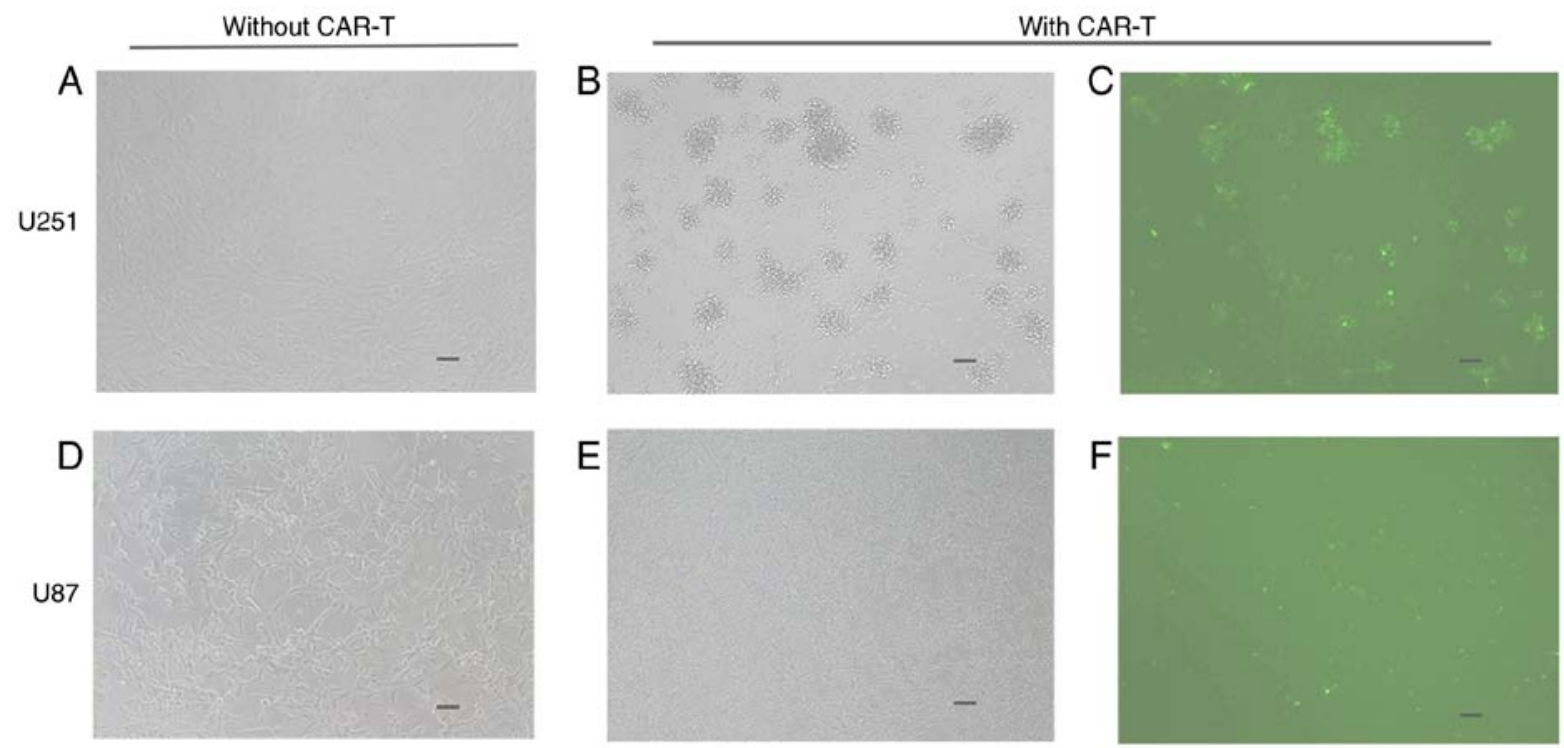

Figure 3. Targeting of anti-HER2 CAR-T cells to HER2-positive glioblastoma cells. HER2-positive U251 glioblastoma cells were co-cultured with anti-HER2 CAR-T cells at effector:target ratio 4:1 for $24 \mathrm{~h}$, observed under a fluorescence microscope in (B) visible light and (C) fluorescence. U251 cells that were not co-cultured with CAR-T cells were examined in (A) visible light concurrently as a control of (B). (D-F) The HER2-negative U87 glioblastoma cells were considered as a negative control (scale bar, $100 \mu \mathrm{m}$ ). HER2, human epidermal growth factor 2; CAR, chimeric antigen receptor; CAR-T, T cells transduced with CAR.
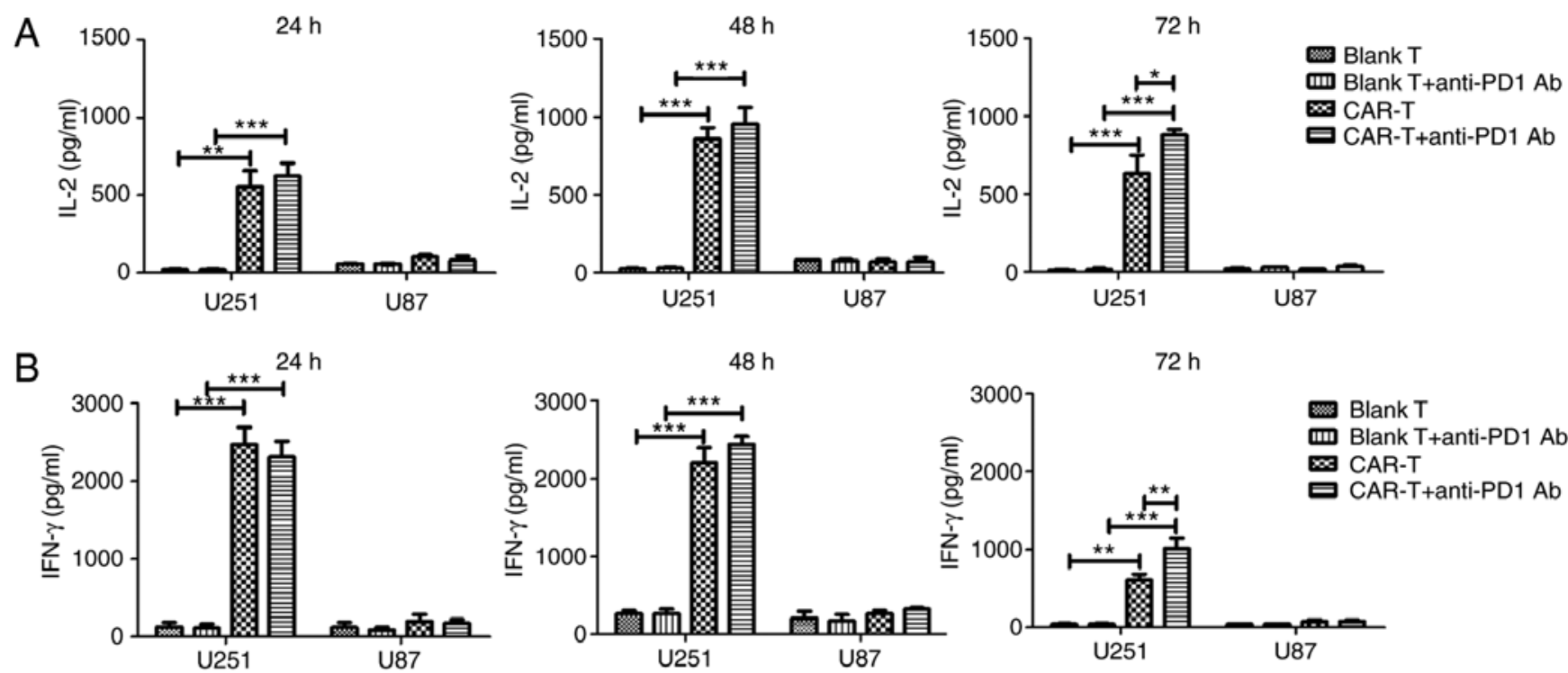

Figure 4. Cytokine secretion of anti-HER2 CAR-T cells. After anti-HER2 CAR-T cells (effector cells) (or in combination with anti-PD1 antibody) were co-cultured with HER2 ${ }^{+}$glioblastoma cells U251 (target cells) at an effector to target ratio of 4:1 for 24,48 or $72 \mathrm{~h}$, supernatant was collected. ELISA kits for detecting (A) IL-2 and (B) IFN- $\gamma$ were used to analyze supernatants. Supernatants from the co-culture of anti-HER2 CAR-T cells with HER2- glioblastoma cells U87, as well as from the co-culture of (untransduced) blank T cells (or in combination with anti-PD1 antibody) with HER $2^{+}$glioblastoma cells U251 were used as negative controls. Three determinations were performed and the mean values are presented. For (A) IL-2 or (B) IFN- $\gamma$ secretion, the CAR-T group was compared with the blank T group, or the CAR-T in combination with anti-PD1 Ab group was compared with blank T in combination with anti-PD1 Ab group, or the CAR-T in combination with anti-PD1 Ab group was compared with the CAR-T group. ${ }^{*} \mathrm{P}<0.05 ;{ }^{* *} \mathrm{P}<0.01 ;{ }^{* * *} \mathrm{P}<0.001$. HER2, human epidermal growth factor 2; CAR, chimeric antigen receptor; CAR-T, T cells transduced with CAR; IL-2, interleukin-2; IFN- $\gamma$, interferon- $\gamma$.

anti-HER2 CAR utilized was second-generation (20). Most CAR-T clinical trials have used second-generation CAR for treating hematological malignancies. To date, there has been no successful clinical trial of anti-HER2 CAR-T cells which has utilized third-generation CAR (https://www.clinicaltrials. gov). In the present study, anti-HER2 CAR was transduced into $\mathrm{T}$ cells by lentiviral vector. To date, there has also been no successful clinical trial for anti-HER2 CAR-T cells which used lentiviral vectors (https://www.clinicaltrials.gov).
The present study demonstrated that third generation anti-HER2 CAR-T cells (with co-stimulatory molecules CD28 and 4-1BB/CD137) are able to eliminate HER2-positive malignant glioblastoma cells both specifically and efficiently. The specialty for binding of CAR to antigen can be affected by the affinity of the selected scFv. The present study used the anti-HER 2 scFv from antibody 4D5 for CAR construction, and the CAR-T cells demonstrated HER2-specific tumor cell lysis in vitro. A functionality assay revealed that these redirected 
A

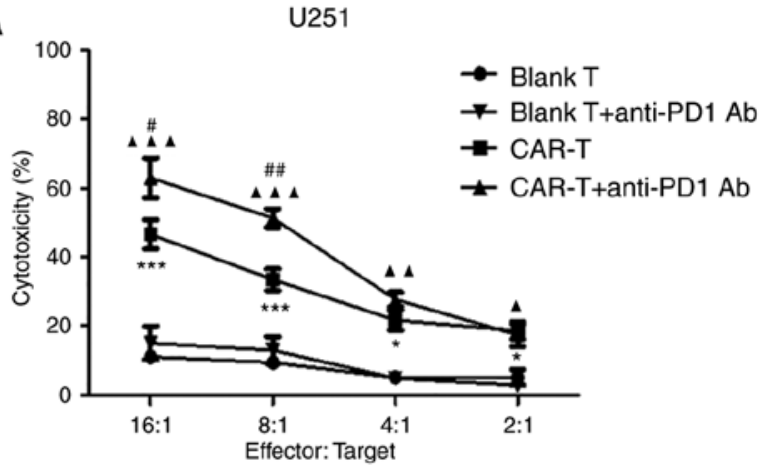

B

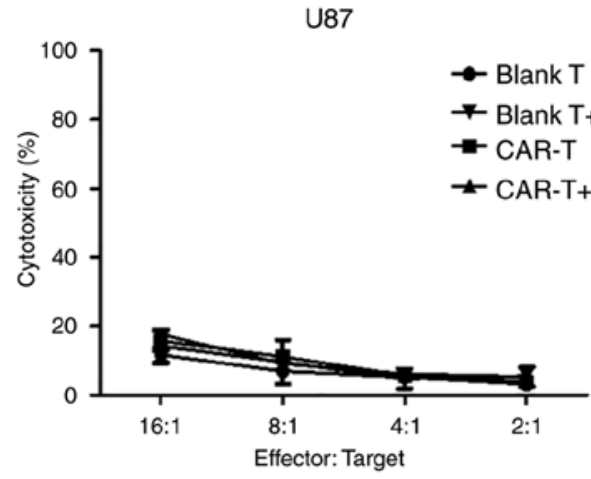

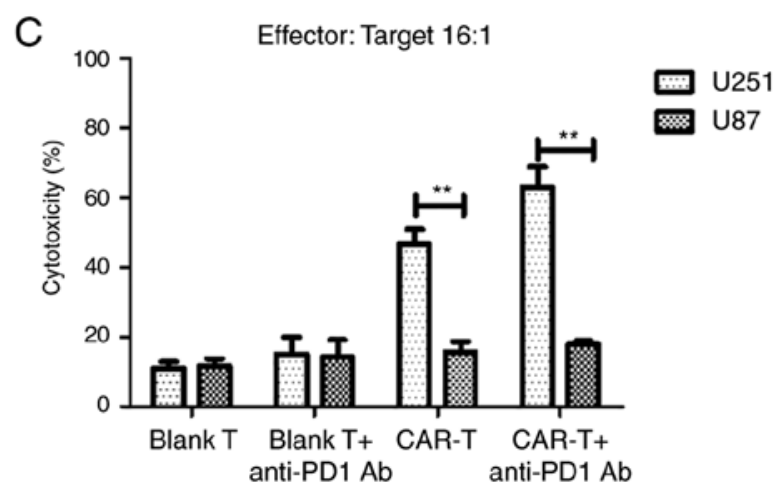

Figure 5. The cytotoxicity of anti-HER2 CAR-T cells (or in combination with anti-PD1 antibody) against HER2-positive glioblastoma cells. (A) After anti-HER2 CAR-T cells (effector cells) were co-cultured with HER2 ${ }^{+}$glioblastoma cells U251 (target cells) (or in combination with anti-PD1 antibody) at different ratios of E:T for $18 \mathrm{~h}$, supernatant was detected using a cytotoxicity LDH detection kit for LDH released from lysed target cells. Cytotoxicity (\%) was calculated. At E:T ratios of $2: 1,4: 1,8: 1$ or $16: 1$ respectively, the cytotoxicity $(\%)$ of the CAR-T group was compared with the Blank T group, ${ }^{*} \mathrm{P}<0.05,{ }^{* * *} \mathrm{P}<0.001$;

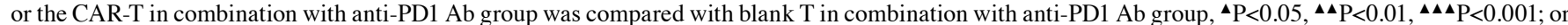
the CAR-T in combination with anti-PD1 Ab group was compared with the CAR-T group, ${ }^{\#} \mathrm{P}<0.05$, ${ }^{\#} \mathrm{P}<0.01$. (B) As a negative control, anti-HER2 CAR-T cells were co-cultured with HER2- glioblastoma cells U87. (C) At E:T ratio 16:1, the cytotoxicity (\%) of the U251 group was compared with the U87 group, ${ }^{* *}<0.01$. Three determinations were performed and the mean values are presented. HER2, human epidermal growth factor 2; CAR, chimeric antigen receptor; CAR-T, T cells transduced with CAR; PD1, programmed death 1; E:T, effector to target; LDH, lactate dehydrogenase.

T cells could secret both cytokine IL-2 and IFN- $\gamma$ as well as exert efficient cytotoxic T cell response in an HER2-specific manner. Major progress has been made since the introduction of co-stimulatory signaling into the architecture of CAR. Given the in-depth understanding of co-stimulatory signaling in $\mathrm{T}$ cell immune response, several co-stimulatory molecules (including CD28, 4-1BB/CD137, CD27, OX40) were embedded in the CAR, following which their roles in coordinating antitumor immunity were explored (30). When compared with other co-stimulatory molecules, CD28 was more effective at enhancing IL-2 production, improving clonal expansion and maintaining persistence of CAR-T cells. CD28 used with $4-1 \mathrm{BB} / \mathrm{CD} 137$ signaling is more effective than CD28 alone in terms of cytotoxicity and IFN- $\gamma$ production (31). The inclusion of the CD137 co-stimulatory molecule in CAR appears more key than CD28. Finney et al demonstrated that CD137 in the CAR induced a maximal effect on target cell lysis (32).

The present results revealed that the presence of anti-PD1 antibody is critical for enhancing $\mathrm{T}$ cell response to tumor cells. CAR-T cells, in combination with anti-PD1 antibody, could release more IL-2 and IFN- $\gamma$ after targeting tumor cells, and were more efficient for eliminating tumor cells when compared with CAR-T cells alone. The main function of PD1 is to reduce the sensitivity of cancer patients to T cell-mediated anti-tumor immune response. Blocking PD1 signaling can rescue exhausted $\mathrm{T}$ lymphocytes and is an effective treatment for cancer. In recent years, checkpoint inhibitors for the PD1/PDLl checkpoint pathway have demonstrated significant antitumor effects in advanced melanoma, classic Hodgkin's lymphoma, non-small cell lung cancer (NSCLC) and other conditions (33-36). Research has suggested that CAR-T cell therapy (including targeting CD19, PSMA) in combination with PD1 blockade is an ideal therapy combination for the treatment of solid tumors $(37,38)$.

Liu et al confirmed that treatment of mice that have large, established solid tumors with PD1-CD28 CAR-T cells led to a significant regression in tumor volume due to to enhanced CAR tumor-infiltrating lymphocyte (TIL) infiltrate, decreased susceptibility to tumor-induced hypofunction and attenuation of inhibitory receptor expression when compared to treatments with CAR-T cells alone or PD1 antibodies (39). Cherkassky et al have reported that anti-MSLN CAR-T cells used in combination with PD1 blockade could enhance the function of CAR-T cells in PDL1 ${ }^{+}$tumor tissues, and may lead to a longer, tumor-free survival period for cancer patients (40).

Future research will verify the in vivo effect of third generation anti-HER2 CAR-T cells in combination with anti-PD1 antibody via a glioblastoma animal model and thus ascertain whether this therapy can prolong the survival of a glioblastoma animal model or even achieve tumor-free survival.

In summary, the present study demonstrated that third generation anti-HER2 CAR-T cells are able to eliminate 
malignant glioblastoma cells specifically and efficiently. Blocking PD1 immuno-suppression can increase activation of CAR-T cells after activation by targeting antigens. This study revealed that, when used in combination with anti-PD1 antibody, anti-HER2 CAR-T cells have a greater therapeutic activity against $\mathrm{HER} 2^{+} / \mathrm{PDL1}^{+}$malignant glioblastoma cells when compared with anti-HER2 CAR-T cells alone.

\section{Acknowledgments}

Not applicable.

\section{Funding}

The present research was supported by grants from the National Natural Science Foundation of China [grant no. 81572980] and the Wenzhou Science and Technology Bureau of China [grant no. H20170001] awarded to HL, as well as from the National Natural Science Foundation of China [grant no. 81772819] awarded to HG.

\section{Availability of data and materials}

Datasets used and analyzed during the current study are available from the corresponding authors on reasonable request.

\section{Authors' contributions}

WY and LS undertook the research, analyzed the data and wrote the paper. SB, PL and JC helped to perform the research and experiments. HL and HG designed the research, wrote and revised the paper. All of the authors read and approved the final manuscript and agree to be for all aspects of the work in ensuring that questions related to the accuracy or integrity of any part of the work are appropriately investigated and resolved. All authors read and approved the final manuscript.

\section{Ethics approval and consent to participate}

The present study was approved by the Ethics Committee of Wenzhou Medical University (Wenzhou, China). Peripheral blood materials used in this study were obtained from healthy donors who provided informed consent.

\section{Patient consent for publication}

Not applicable.

\section{Competing interests}

The authors declare that they have no competing interests.

\section{References}

1. Reardon DA and Mitchell DA: The development of dendritic cell vaccine-based immunotherapies for glioblastoma. Semin Immunopathol 39: 225-239, 2017.

2. Ohba S and Hirose Y: Current and Future Drug Treatments for Glioblastomas. Curr Med Chem 23: 4309-4316, 2016.
3. Batash R, Asna N, Schaffer P, Francis N and Schaffer M: Glioblastoma multiforme, diagnosis and treatment; recent literature review. Curr Med Chem 24: 3002-3009, 2017.

4. Hao L, Li T, Chang LJ and Chen X: Adoptive immunotherapy for B-cell malignancies using CD19-targeted chimeric antigen receptor T-cells: A systematic review of efficacy and safety. Curr Med Chem: Aug 1, 2017 (Epub ahead of print). doi: 10.2174/092 9867324666170801101842

5. Jacoby E, Bielorai B, Avigdor A, Itzhaki O, Hutt D, Nussboim V, Meir A, Kubi A, Levy M, Zikich D, et al: Locally produced CD19 CAR T cells leading to clinical remissions in medullary and extramedullary relapsed acute lymphoblastic leukemia. Am J Hematol 93: 1485-1492, 2018.

6. Wang D, Shi R, Wang Q and Li J: Extramedullary relapse of acute lymphoblastic leukemia after allogeneic hematopoietic stem cell transplantation treated by CAR T-cell therapy: A case report. Onco Targets Ther 11: 6327-6332, 2018.

7. Jena B, Dotti G and Cooper LJ: Redirecting T-cell specificity by introducing a tumor-specific chimeric antigen receptor. Blood 116: 1035-1044, 2010.

8. Potti A, Forseen SE, Koka VK, Pervez H, Koch M, Fraiman G, Mehdi SA and Levitt R: Determination of HER-2/neu overexpression and clinical predictors of survival in a cohort of 347 patients with primary malignant brain tumors. Cancer Invest 22: 537-544, 2004

9. Koka V, Potti A, Forseen SE, Pervez H, Fraiman GN, Koch M and Levitt R: Role of Her-2/neu overexpression and clinical determinants of early mortality in glioblastoma multiforme. Am J Clin Oncol 26: 332-335, 2003.

10. Seliger B, Rongcun Y, Atkins D, Hammers S, Huber C, Storkel S and Kiessling R: HER-2/neu is expressed in human renal cell carcinoma at heterogeneous levels independently of tumor grading and staging and can be recognized by HLA-A2.1-restricted cytotoxic T lymphocytes. Int J Cancer 87: 349-359, 2000.

11. Wang S, Saboorian MH, Frenkel E, Hynan L, Gokaslan ST and Ashfaq R: Laboratory assessment of the status of Her- $2 /$ neu protein and oncogene in breast cancer specimens: Comparison of immunohistochemistry assay with fluorescence in situ hybridisation assays. J Clin Pathol 53: 374-381, 2000.

12. Li BT, Ross DS, Aisner DL, Chaft JE, Hsu M, Kako SL, Kris MG, Varella-Garcia $M$ and Arcila ME: HER2 amplification and HER2 mutation are distinct molecular targets in lung cancers. J Thorac Oncol 11: 414-419, 2016.

13. Press MF, Cordon-Cardo C and Slamon DJ: Expression of the HER-2/neu proto-oncogene in normal human adult and fetal tissues. Oncogene 5: 953-962, 1990.

14. Whilding LM and Maher J: ErbB-targeted CAR T-cell immunotherapy of cancer. Immunotherapy 7: 229-241, 2015.

15. Nowakowska P, Romanski A, Miller N, Odendahl M, Bonig H, Zhang C, Seifried E, Wels WS and Tonn T: Clinical grade manufacturing of genetically modified, CAR-expressing NK-92 cells for the treatment of ErbB2-positive malignancies. Cancer Immunol Immunother 67: 25-38, 2018.

16. Liu G, Ying H, Zeng G, Wheeler CJ, Black KL and Yu JS: HER-2, gp100, and MAGE-1 are expressed in human glioblastoma and recognized by cytotoxic T cells. Cancer Res 64: 4980-4986, 2004.

17. Ahmed N, Salsman VS, Kew Y, Shaffer D, Powell S, Zhang YJ, Grossman RG, Heslop HE and Gottschalk S: HER2-specific $\mathrm{T}$ cells target primary glioblastoma stem cells and induce regression of autologous experimental tumors. Clin Cancer Res 16: 474-485, 2010.

18. Thomas CY, Chouinard M, Cox M, Parsons S, Stallings-Mann M, Garcia R, Jove R and Wharen R: Spontaneous activation and signaling by overexpressed epidermal growth factor receptors in glioblastoma cells. Int J Cancer 104: 19-27, 2003.

19. Andersson U, Guo D, Malmer B, Bergenheim AT, Brannstrom T, Hedman $\mathrm{H}$ and Henriksson R: Epidermal growth factor receptor family (EGFR, ErbB2-4) in gliomas and meningiomas. Acta Neuropathol 108: 135-142, 2004.

20. Kuramitsu S, Yamamichi A, Ohka F, Motomura K, Hara M and Natsume A: Adoptive immunotherapy for the treatment of glioblastoma: Progress and possibilities. Immunotherapy 8: 1393-1404, 2016.

21. Tlsty TD and Coussens LM: Tumor stroma and regulation of cancer development. Annu Rev Pathol 1: 119-150, 2006.

22. Denko NC: Hypoxia, HIF1 and glucose metabolism in the solid tumour. Nat Rev Cancer 8: 705-713, 2008.

23. Zheng Y, Zha Y and Gajewski TF: Molecular regulation of T-cell anergy. EMBO Rep 9: 50-55, 2008. 
24. Gajewski TF, Schreiber H and Fu YX: Innate and adaptive immune cells in the tumor microenvironment. Nat Immunol 14: 1014-1022, 2013.

25. Al-Zoughbi W, Huang J, Paramasivan GS, Till H, Pichler M, Guertl-Lackner B and Hoefler G: Tumor macroenvironment and metabolism. Semin Oncol 41: 281-295, 2014.

26. Zou W and Chen L: Inhibitory B7-family molecules in the tumour microenvironment. Nat Rev Immunol 8: 467-477, 2008.

27. Keir ME, Butte MJ, Freeman GJ and Sharpe AH: PD-1 and its ligands in tolerance and immunity. Annu Rev Immunol 26 : 677-704, 2008.

28. Yuan W, Chen J, Cao Y, Yang L, Shen L, Bian Q, Bin S, Li P, Cao J, Fang H, et al: Comparative analysis and optimization of protocols for producing recombinant lentivirus carrying the anti-Her2 chimeric antigen receptor gene. J Gene Med 20: e3027, 2018.

29. Wang C, Hu W, Shen L, Dou R, Zhao S, Shan D, Yu K, Huang R and $\mathrm{Li} \mathrm{H}$ : Adoptive antitumor immunotherapy in vitro and in vivo using genetically activated erbB2-specific $\mathrm{T}$ cells J Immunother 37: 351-359, 2014.

30. Zhong XS, Matsushita M, Plotkin J, Riviere I and Sadelain M: Chimeric antigen receptors combining 4-1BB and CD28 signaling domains augment PI3kinase/AKT/Bcl-XL activation and $\mathrm{CD}^{+} \mathrm{T}$ cell-mediated tumor eradication. Mol Ther 18 413-420, 2010.

31. Brentjens RJ, Santos E, Nikhamin Y, Yeh R, Matsushita M, La Perle K, Quintas-Cardama A, Larson SM and Sadelain M: Genetically targeted $\mathrm{T}$ cells eradicate systemic acute lymphoblastic leukemia xenografts. Clin Cancer Res 13: 5426-5435, 2007.

32. Finney HM, Akbar AN and Lawson AD: Activation of resting human primary $\mathrm{T}$ cells with chimeric receptors: Costimulation from CD28, inducible costimulator, CD134, and CD137 in series with signals from the TCR zeta chain. J Immunol 172: 104-113, 2004.
33. Roger A, Finet A, Boru B, Beauchet A, Mazeron JJ, Otzmeguine Y, Blom A, Longvert C, de Maleissye MF, Funck-Brentano E and Saiag P: Efficacy of combined hypo-fractionated radiotherapy and anti-PD-1 monotherapy in difficult-to-treat advanced melanoma patients. Oncoimmunology 7: e1442166, 2018.

34. Taquin H, Fontas E, Massol O, Chevallier P, Balloti R, Beranger G, Lacour JP, Passeron T and Montaudie H: Efficacy and safety data for checkpoint inhibitors in advanced melanoma under real-life conditions: A monocentric study conducted in Nice from 2010 to 2016. Ann Dermatol Venereol 145: 649-658, 2018.

35. Wang Y, Zhang X, Yang L, Xue J and Hu G: Blockade of CCL2 enhances immunotherapeutic effect of anti-PD1 in lung cancer. J Bone Oncol 11: 27-32,2018.

36. Zibetti Dal Molin G, Abrahao CM, Coleman RL and Maluf FC Response to pembrolizumab in a heavily treated patient with metastatic ovarian carcinosarcoma. Gynecol Oncol Res Pract 5: 6, 2018.

37. Li S, Siriwon N, Zhang X, Yang S, Jin T, He F, Kim YJ, Mac J, Lu Z, Wang S, et al: Enhanced cancer immunotherapy by chimeric antigen receptor-modified t cells engineered to secrete checkpoint inhibitors. Clin Cancer Res 23: 6982-6992, 2017.

38. Serganova I, Moroz E, Cohen I, Moroz M, Mane M, Zurita J, Shenker L, Ponomarev V and Blasberg R: Enhancement of PSMA-directed CAR adoptive immunotherapy by PD-1/PD-L1 blockade. Mol Ther Oncolytics 4: 41-54, 2017.

39. Liu X, Ranganathan R, Jiang S, Fang C, Sun J, Kim S, Newick K, Lo A, June CH, Zhao Y and Moon EK: A chimeric switch-receptor targeting PD1 augments the efficacy of second-generation car t cells in advanced solid tumors. Cancer Res 76: 1578-1590, 2016.

40. Cherkassky L, Morello A, Villena-Vargas J, Feng Y, Dimitrov DS, Jones DR, Sadelain M and Adusumilli PS: Human CAR T cells with cell-intrinsic PD-1 checkpoint blockade resist tumor-mediated inhibition. J Clin Invest 126: 3130-3144, 2016. 